\title{
Systematic review and meta-analysis of the efficacy of general anesthesia combined with a thoracic nerve block in modified breast cancer surgery
}

\author{
Juan Liao ${ }^{1 \#}$, Meiting $\mathrm{Li}^{2 \#}$, Jiaqi Gan ${ }^{3,4 \#}$, Jie Xiao ${ }^{2,4}$, Guilin Xiang ${ }^{2,4}$, Xizhi Ding ${ }^{2}$, Rong Jiang ${ }^{2}$, Peng $\mathrm{Li}^{2}$ \\ ${ }^{1}$ Department of Stomatology, Sichuan Provincial People's Hospital, University of Electronic Science and Technology of China, Chengdu, China; \\ ${ }^{2}$ Department of Anesthesiology, Sichuan Provincial People's Hospital, University of Electronic Science and Technology of China, Chengdu, China; \\ ${ }^{3}$ Department of Anesthesiology, Sichuan Provincial Maternity and Child Health Care Hospital, Chengdu, China; ${ }^{4} \mathrm{Chengdu}$ Medical College, \\ Chengdu, China \\ Contributions: (I) Conception and design: J Liao, M Li, J Gan, R Jiang, P Li; (II) Administrative support: J Xiao; (III) Provision of study materials or \\ patients: G Xiang, X Ding, J Gan, R Jiang, P Li; (IV) Collection and assembly of data: J Liao, M Li, J Gan, R Jiang, J Xiao; (V) Data analysis and \\ interpretation: All authors; (VI) Manuscript writing: All authors; (VII) Final approval of manuscript: All authors. \\ "These authors contributed equally to the work. \\ Correspondence to: Peng Li; Rong Jiang. Department of Anesthesiology, Sichuan Provincial People's Hospital, University of Electronic Science and \\ Technology of China, Chengdu 610072, China. Email: lipengmazui@qq.com; anthea_365@163.com.
}

Background: Breast cancer is a malignant tumor disease that poses a significant threat to women's health. In recent years, the incidence of breast cancer in China has been increasing. This report aims to explore the effects of general anesthesia combined with a thoracic nerve block in modified breast cancer surgery.

Methods: A computer-based search of PubMed, Web of Science, Embase, and the Cochrane Library was performed to identify randomized controlled studies on breast cancer, general anesthesia combined with a thoracic nerve block, modified breast cancer surgery, and other breast cancer treatments. Further search criteria included postoperative pain score, postoperative morphine equivalents given 24 hours after surgery, and operation duration. After an initial selection process, the studies were evaluated using the Jadad scale and the Cochrane Handbook for Systematic Reviews of Interventions to assess their suitability for inclusion in the subsequent meta-analysis of the experimental data, which was carried out using RevMan 5.3.

Results: A total of 8 studies comprising a total of 624 patients were selected for inclusion in this report. According to the meta-analysis, the analytical structure of the thoracic nerve group and the control group had a mean difference (MD) of -1.27 [95\% confidence interval (CI): -1.68 to -0.86 ], the structure of the statistical test was $\mathrm{Z}=6.08(\mathrm{P}<0.00001)$, the $\mathrm{MD}$ of the total analysis structure of morphine equivalents was -2.71 (95\% CI: -4.98 to -0.44 ), and the statistical test structure was $\mathrm{Z}=2.34$ ( $\mathrm{P}=0.02)$.

Discussion: General anesthesia combined with a thoracic nerve block in breast cancer surgery may effectively improve postoperative pain in patients and reduce the need for analgesic drugs. However, the outcome indicators included in this study are not sufficient. It is necessary to increase both the sample size and the number of outcome indicators to provide further theoretical evidence for the subsequent application of thoracic nerve block in modified breast cancer surgery.

Keywords: General anesthesia; complex thoracic nerve block; postmastectomy pain syndrome (PMPS); modified breast cancer surgery

Submitted Sep 13, 2021. Accepted for publication Nov 16, 2021.

doi: 10.21037 /gs-21-719

View this article at: https://dx.doi.org/10.21037/gs-21-719 


\section{Introduction}

Breast cancer is an exceedingly common form of malignant tumor disease and is considered to be one of the greatest threats to women's safety and health. According to relevant data, breast cancer ranks first among all malignant tumors in women (1-3). The incidence of breast cancer in China is increasing year by year and becoming more prominent in areas with underdeveloped medical services. Early detection and treatment of breast cancer can greatly improve the survival rate of patients, and the primary treatment for early breast cancer patients is surgical resection. Postmastectomy pain syndrome (PMPS) is a chronic neuropathic pain syndrome that can occur after a mastectomy (4-6). In current cases, the syndrome produces pain that lasts for at least 3 months although it has been known to last up to 9 years. The etiology and mechanism of PMPS are still unclear. The current mainstream view is that intercostal nerve damage is caused after mastectomy, which in turn causes intercostal neuralgia. The type of surgery, radiotherapy, chemotherapy, age, perioperative treatment, and tumor size are all risk factors for PMPS (7). Some patients go on to develop PMPS, partly because they are not well-informed about the condition. Most patients do not come forward for formal diagnosis and treatment at the onset of pain, choosing instead to self-manage intense postoperative pain (8). Thus, research and awareness of postoperative complications need to be expanded in China.

Related studies have found that the occurrence of PMPS is predictable and can be effectively prevented with the timely detection and management of risk factors in clinical practice. The development of PMPS after surgery is generally influenced by several factors, and data show that PMPS is inextricably linked to the severity of acute postoperative pain (9-11). If acute postoperative pain is not treated, it is highly likely to develop into PMPS. Currently, opioid analgesics are the most frequently prescribed for the clinical treatment of PMPS. Both the analgesic effect and adverse reactions to these drugs are proportional to the dosage. If a favorable analgesic effect is to be achieved, the dosage should be increased; however, the incidence of adverse reactions, such as vomiting and respiratory depression, will also increase accordingly (12). Studies indicate that a perioperative nerve block combined with opioids can effectively lower the required dosage of the latter, reducing adverse reactions while maintaining a strong analgesic effect (13). Intercostal nerve block has the advantages of less trauma, low cost and obvious effect. The completion of puncture and drug delivery with the assistance of ultrasound can significantly improve the success rate and safety of nerve block, and effectively reduce the occurrence of complications (14). Researchers have found that an intercostal nerve block in breast cancer surgery can have a powerful analgesic effect, effectively reducing the use of postoperative analgesic drugs and adverse reactions (15).

As medical technology has developed in recent years, thoracic nerve blocks have become more widely used for breast cancer surgery, providing the advantages of a simpler, shorter, and lower-risk operation. The thoracic type-1 nerve block was first proposed as early as $2011(16,17)$ and can completely anesthetize the medial and lateral thoracic nerves, providing a good anesthetic effect on the medial side of the chest wall. In addition, a thoracic type- 2 nerve block can provide axillary anesthesia, mainly with long thoracic nerve and intercostal nerve blocks. The combination of the two types of nerve block can provide good perioperative anesthesia and postoperative analgesia for patients $(18,19)$ and constitutes an excellent treatment for acute pain in breast cancer patients.

At present, there are few systematic reviews about the effects of general anesthesia and nerve block anesthesia on postoperative analgesia and prognosis of breast cancer patients. In order to provide more research evidence. This report analyzes and explores the efficacy of general anesthesia combined with a thoracic nerve block in modified breast cancer surgery and provides a reference for the future clinical treatment and prognosis of breast cancer. We present the following article in accordance with the PRISMA reporting checklist (available at https://dx.doi. org/10.21037/gs-21-719).

\section{Methods}

\section{Study identification and selection}

A computer-based search of English-language databases including PubMed, Web of Science, Embase, and the Cochrane Library was carried out. The date parameter for the search was set from January 2000 to September 2020. Search terms included "general anesthesia", "compound pectoral nerve block", "postmastectomy pain syndrome", and "modified operation for breast cancer", among others.

\section{Inclusion and exclusion criteria}

For inclusion in this meta-analysis, studies were required 
to have the following characteristics: (I) a focus on general anesthesia combined with a thoracic nerve block in modified breast cancer; (II) a direct or indirect evaluation of patient experience after breast cancer surgery; and (III) a sample size of at least 15 patients.

Studies were excluded in the following cases: (I) if they repeated publication of a set of data from another study; (II) if they were a review, a conference report, an experience lecture, a case report; (III) if the research was unrelated to the topic of this report; (IV) if the study did not set a control group, or if the data between the groups are not comparable; or $(\mathrm{V})$ if the outcome indicators were not reported clearly and the results data were incomplete.

\section{Quality assessment}

After the initial selection, two researchers independently read the full texts of the studies and extracted any relevant information. Disagreements or disputes were resolved through discussion or with the assistance of a third investigator. The Jadad scale was used to evaluate the methodological quality of the selected studies and assessed the following parameters: (I) whether the study was a randomized trial; (II) whether the randomization method used was appropriate; (III) whether the study used double blinding; (IV) whether the double-blind method was appropriate; and $(\mathrm{V})$ whether there was a description of patient withdrawals or dropouts. A yes answer received 1 point, and a no received 0 point for a total score out of 5 . A score of less than 2 indicated low-quality research, while a score of greater than 2 indicated high-quality research.

Subsequently, the Cochrane Handbook for Systematic Reviews of Interventions (version 4.2.5) was used to evaluate the risk of bias in each study and assessed the following: (I) whether it was a randomized trial; (II) whether there was allocation concealment; (III) whether blind testing was used; (IV) whether the reported results were complete; $(\mathrm{V})$ whether there was selective reporting of results; and (VI) whether there was any other bias.

\section{Data extraction}

The following data were extracted from the studies: (I) the name of the first author, year of publication, and evaluation results; (II) the number of study participants, experimental design, specific measures, study time, and outcome indicators; (III) the baseline data of patients; and (IV) the indicators of feedback and research quality.

\section{Statistical analysis}

RevMan 5.3, (Cochrane Collaboration), was used for statistical data analysis. First, Peto's method was used to analyze the heterogeneity of the studies with a cutoff point of $\alpha=0.05$ : when $\mathrm{I}^{2}<50 \%$, no heterogeneity in the literature, and the fixed effects model is used for analysis; when $\mathrm{I}^{2}$ is greater than $50 \%$, heterogeneity is considered to be present in literature, and the random effects model is used for analysis. The weighted mean difference (WMD) is used to represent the results of measurement data using the same unit of measurement; otherwise, the standardized mean difference (SMD) is used to represent the results. The results of counting data are expressed by relative risk (RR). All results are expressed here with $95 \%$ confidence interval (CI). A funnel plot was drawn, and the publication bias was evaluated by the symmetry of the funnel plot and concentration of literature to the midline. Sensitivity analysis was used to assess the reliability and stability of the results.

\section{Results}

\section{Literature search results and profile analysis}

A total of 1,662 records were initially retrieved from the database, and 1,207 abstracts were subsequently obtained after duplicates were eliminated. Once the two researchers had read the titles and abstracts, 385 studies that met the inclusion criteria were preselected. After a review of the full text of the studies, those that were found to be not random, repeat publications, or unavailable in full-text were excluded, and 8 studies that met the inclusion criteria were chosen for this review (20-27). A diagram of the literature retrieval process is shown in Figure 1, and the basic characteristics of the studies selected for this report are shown in Table 1.

\section{Bias risk assessment of included literature}

The bias risk assessment tool recommended by the Cochrane Handbook for Systematic Reviews of Interventions was used to evaluate the quality of the included literature, and the results are shown in Figures 2,3. None of the studies were found to have random sequence generation (selection bias), incomplete outcome data (selection bias), or selective 


\section{Identification of studies via databases and registers}

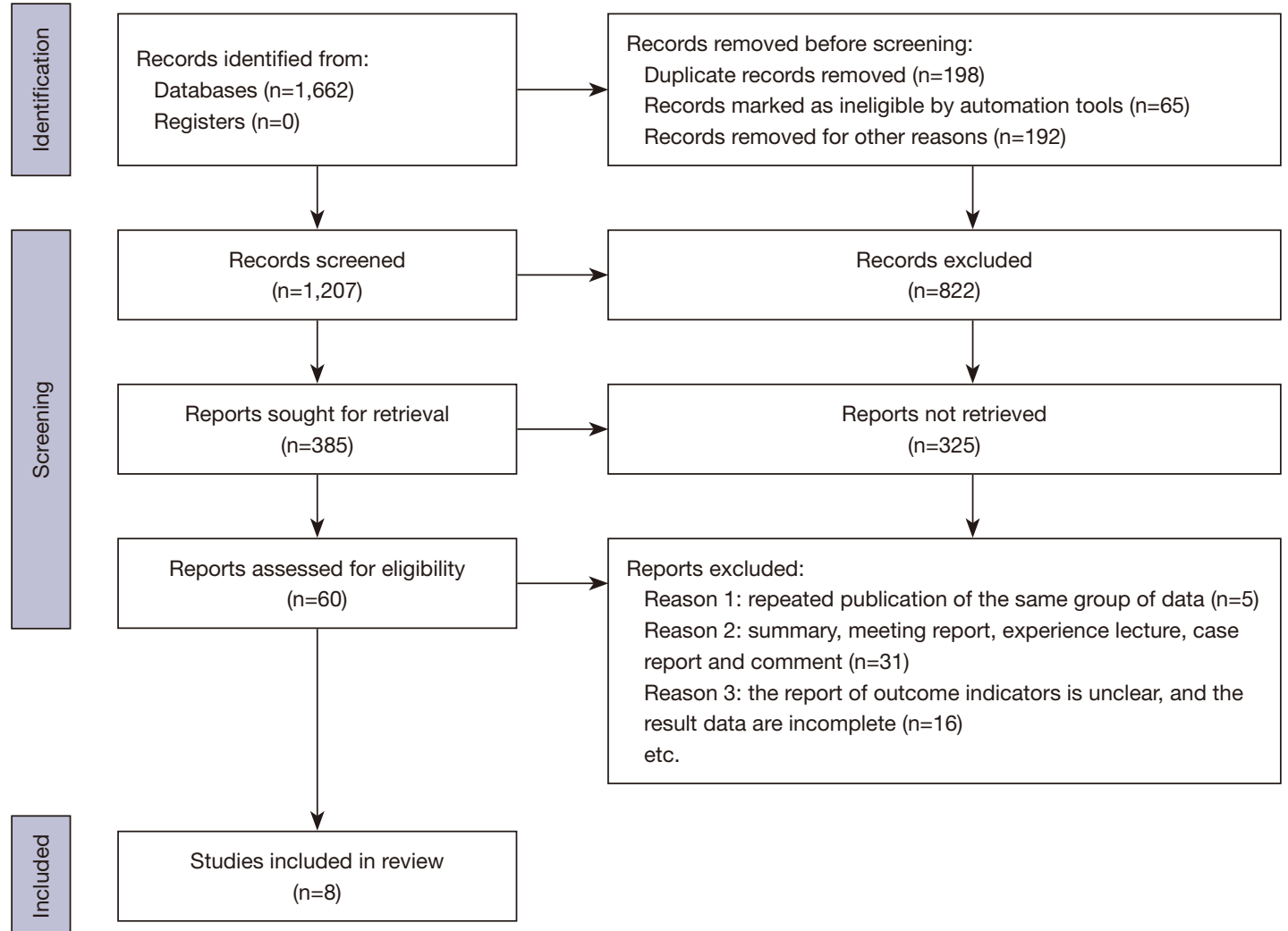

Figure 1 Schematic diagram of the literature screening process.

Table 1 Basic characteristics of the included studies

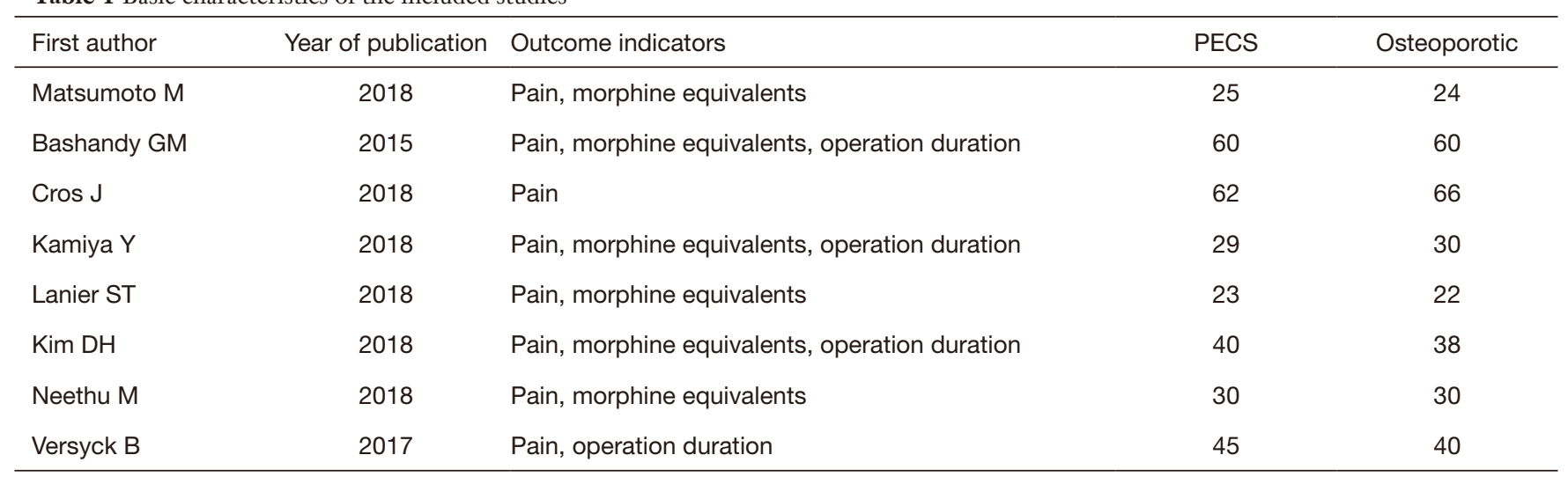

PECS, pectoral nerve.

reporting (reporting bias), and the overall risk of the studies included in this report was low.

The Jadad scale was used to assess the quality of each study, with the results shown in Table 2. The 8 studies included in this report all had a low risk of bias and met the requirements for subsequent analysis. 


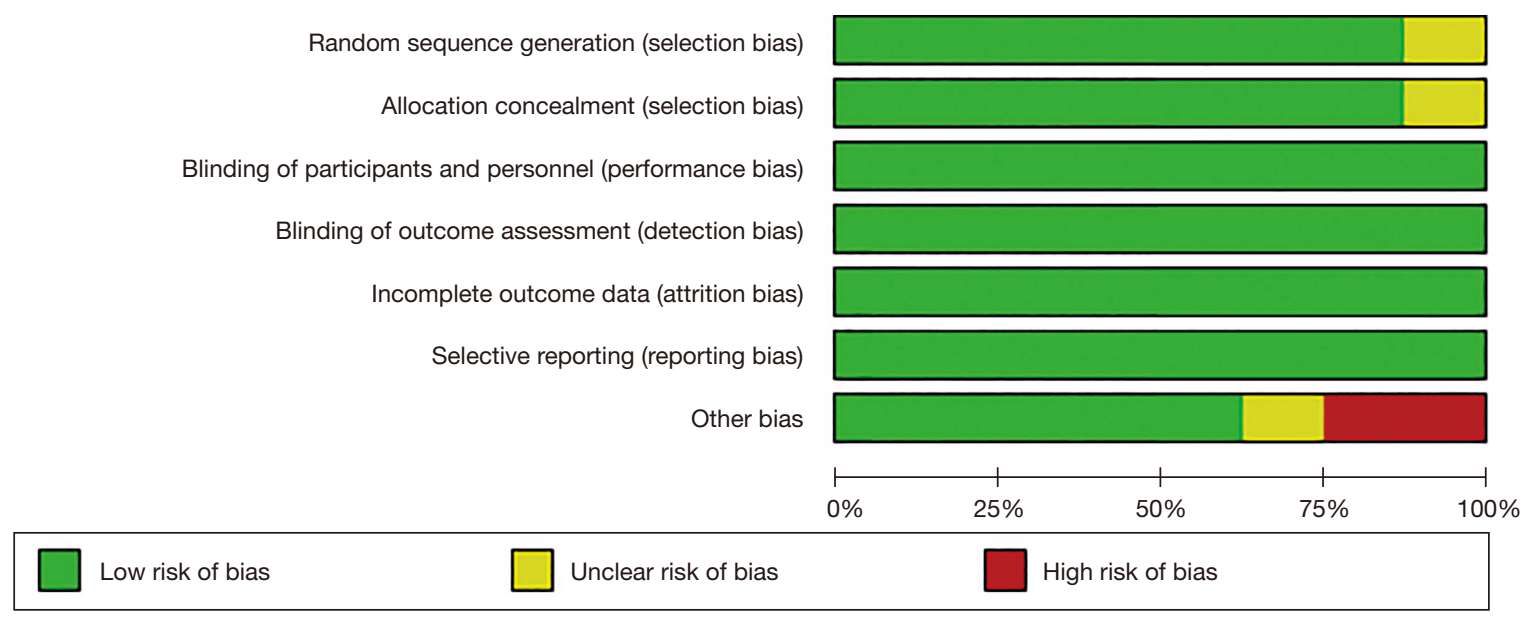

Figure 2 Bar chart of the bias risk assessment of the included literature.

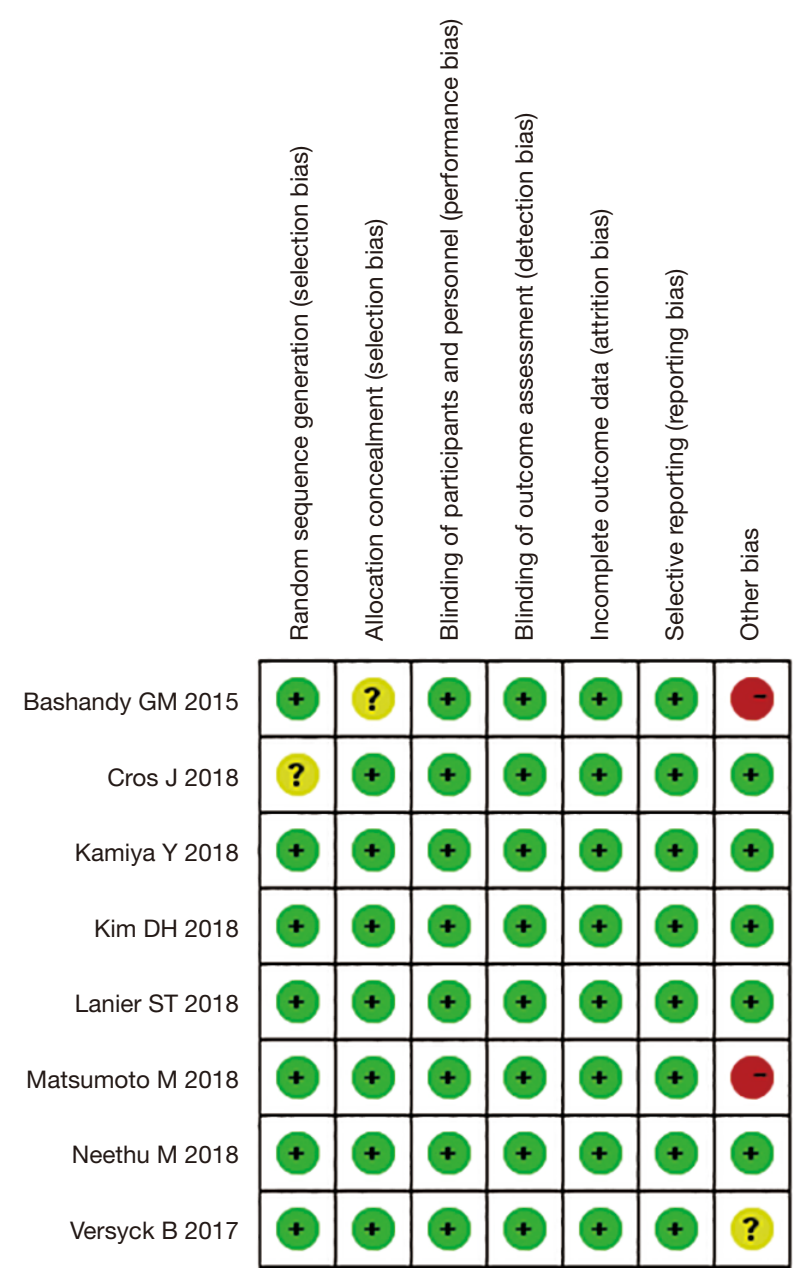

Figure 3 Summary chart of bias risk assessment for the selected studies.

\section{Meta-analysis of pain score}

All 8 studies compared the pain scores of patients in two trial groups: a thoracic nerve group, who received both a general anesthetic and a thoracic nerve block for modified breast surgery; and a control group, who underwent modified breast surgery with only a general anesthetic. The results are shown in Figure 4. The pain scores of the thoracic nerve group and the control group were heterogeneous $\left(\mathrm{I}^{2}=71 \% ; \mathrm{P}=0.0009\right)$. Therefore, the random effects model was used for statistical analysis. The MD of the total pain score of the thoracic nerve group and the control group was -1.27 (95\% CI: -1.68 to -0.86$)$. The statistical test structure was $Z=6.08$ $(\mathrm{P}<0.00001)$. These results indicated that the pain scores of the two groups of patients differed significantly.

\section{Meta-analysis of the total amount of morphine equivalents given to patients after surgery}

Of the selected studies, 6 evaluated in detail the total amount of morphine equivalents given to patients after surgery. These were compared, with results shown in Figure 5. Heterogeneity between the thoracic nerve group and the control group was detected $\left(\mathrm{I}^{2}=98 \% ; \mathrm{P}<0.00001\right)$. Therefore, the random effects model was used for statistical analysis. The MD of morphine equivalents used in the thoracic nerve group and the control group was $-2.71(95 \%$ CI: -4.98 to -0.44$)$. The statistical test structure was $Z=2.34$ $(\mathrm{P}=0.02)$. These results indicated that the total amount of morphine equivalents used after surgery in the two groups 
Table 2 Jadad scale assessment of the quality of the included studies

\begin{tabular}{|c|c|c|c|c|c|c|}
\hline First author & Randomization & Binding & Allocation concealment & Withdrawals and dropouts & $\begin{array}{l}\text { Reason of dropouts } \\
\text { and withdrawals }\end{array}$ & Jadad \\
\hline Matsumoto M & Yes & No & NMT & MT & No & 3 \\
\hline Cros J & Yes & No & NMT & MT & Yes & 3 \\
\hline Kim DH & Yes & No & NMT & MT & No & 3 \\
\hline Neethu M & Yes & No & NMT & MT & No & 3 \\
\hline Versyck B & Yes & No & NMT & MT & No & 3 \\
\hline
\end{tabular}

MT, mentioned; NMT, not mentioned.

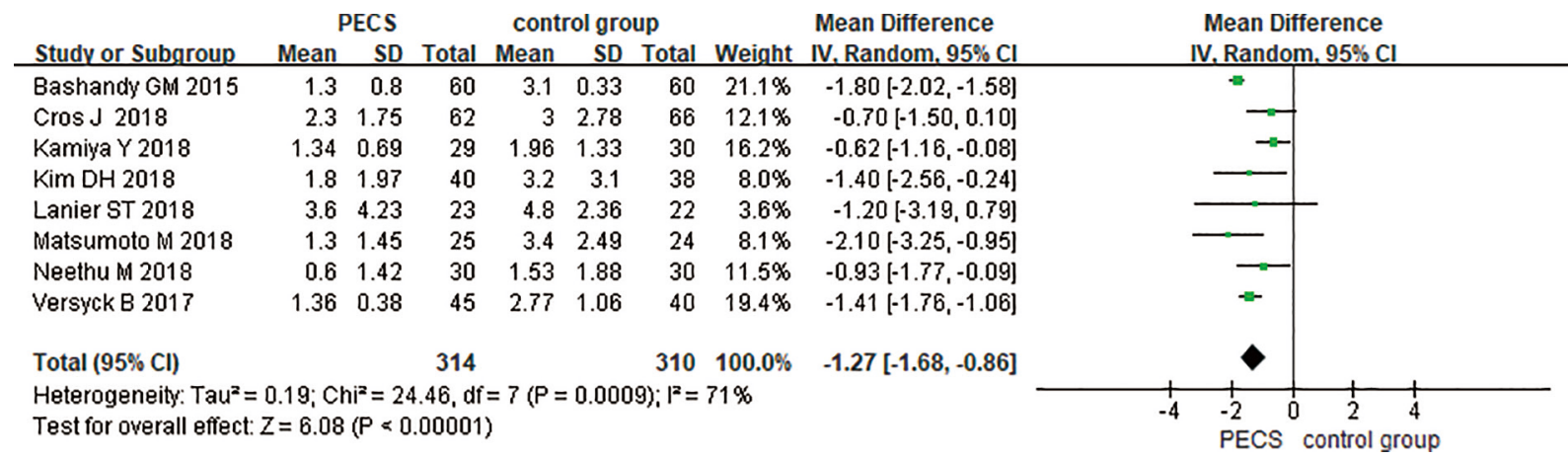

Figure 4 Forest plot of patients pain score after modified breast cancer surgery with general anesthesia combined with a PECS block. PECS, pectoral nerve.

\begin{tabular}{|c|c|c|c|c|c|c|c|c|c|c|c|c|}
\hline \multirow[b]{2}{*}{ Study or Subgroup } & \multicolumn{3}{|c|}{ PECS } & \multicolumn{3}{|c|}{ control group } & \multirow{2}{*}{\multicolumn{2}{|c|}{$\begin{array}{cc}\text { Mean Difference } \\
\text { Weight } & \text { IV. Random. } 95 \% \mathrm{Cl} \\
\end{array}$}} & \multirow{2}{*}{\multicolumn{4}{|c|}{$\begin{array}{c}\text { Mean Difference } \\
\text { IV. Random. } 95 \% \mathrm{Cl}\end{array}$}} \\
\hline & Mean & SD & Total & Mean & SD & Total & & & & & & \\
\hline Bashandy GM 2015 & 2.5 & 1.2 & 60 & 4.3 & 1.8 & 60 & $17.2 \%$ & $-1.80[-2.35,-1.25]$ & & $=$ & & \\
\hline Kamiya Y 2018 & 2.65 & 0.52 & 29 & 3.08 & 0.41 & 30 & $17.3 \%$ & $-0.43[-0.67,-0.19]$ & & & & \\
\hline Kim DH 2018 & 4.31 & 2.92 & 40 & 4.97 & 3.98 & 38 & $16.0 \%$ & $-0.66[-2.22,0.90]$ & & & & \\
\hline Lanier ST 2018 & 8 & 1.52 & 23 & 17 & 1.76 & 22 & $16.8 \%$ & $-9.00[-9.96,-8.04]$ & $\rightarrow-$ & & & \\
\hline Matsumoto M 2018 & 5.8 & 3.52 & 25 & 8.3 & 1.24 & 24 & $16.2 \%$ & $-2.50[-3.97,-1.03]$ & & 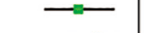 & & \\
\hline Neethu M 2018 & 4.95 & 1.29 & 30 & 6.77 & 3.02 & 30 & $16.6 \%$ & $-1.82[-3.00,-0.64]$ & & - & & \\
\hline Total $(95 \% \mathrm{Cl})$ & & & 207 & & & 204 & $100.0 \%$ & $-2.71[-4.98,-0.44]$ & & & & \\
\hline $\begin{array}{l}\text { Heterogeneity: Tauz } \\
\text { Test for overall effec }\end{array}$ & $\begin{array}{l}8.74 ; \mathrm{Cl} \\
\mathrm{Z}=2.34\end{array}$ & $\begin{aligned} \mathrm{hi}^{2} & =30 \\
(\mathrm{P} & =0\end{aligned}$ & $10.29, d$ & $f=5$ & 0.00 & & $=98 \%$ & & -10 & PECS ${ }^{0}$ & control gro & 5 \\
\hline
\end{tabular}

Figure 5 Forest plot of the total amount of morphine equivalents given to patients after surgery. PECS, pectoral nerve.

differed significantly.

\section{Meta-analysis of operation duration}

Of the studies included in this review, 4 provide a detailed assessment of the duration of the operation. These were compared, with results shown in Figure 6. No heterogeneity was detected between the thoracic nerve group and the control group $\left(\mathrm{I}^{2}=0 \% ; \mathrm{P}=0.71\right)$. Therefore, a fixed effects model was used for statistical analysis. The MD of operation duration of the thoracic nerve group and the control group was 2.11 (95\% CI: -2.35 to 6.58$)$. The statistical test 


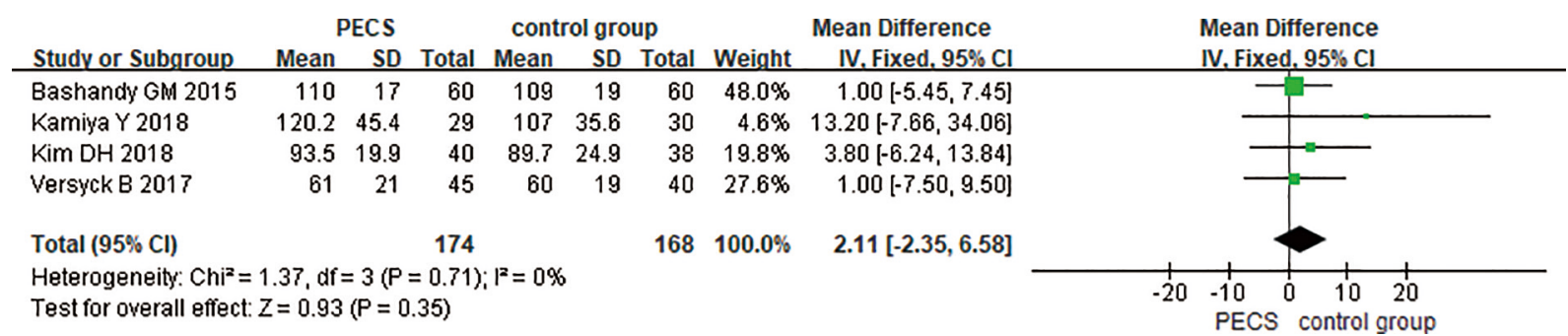

Figure 6 Forest plot of operation duration. PECS, pectoral nerve.
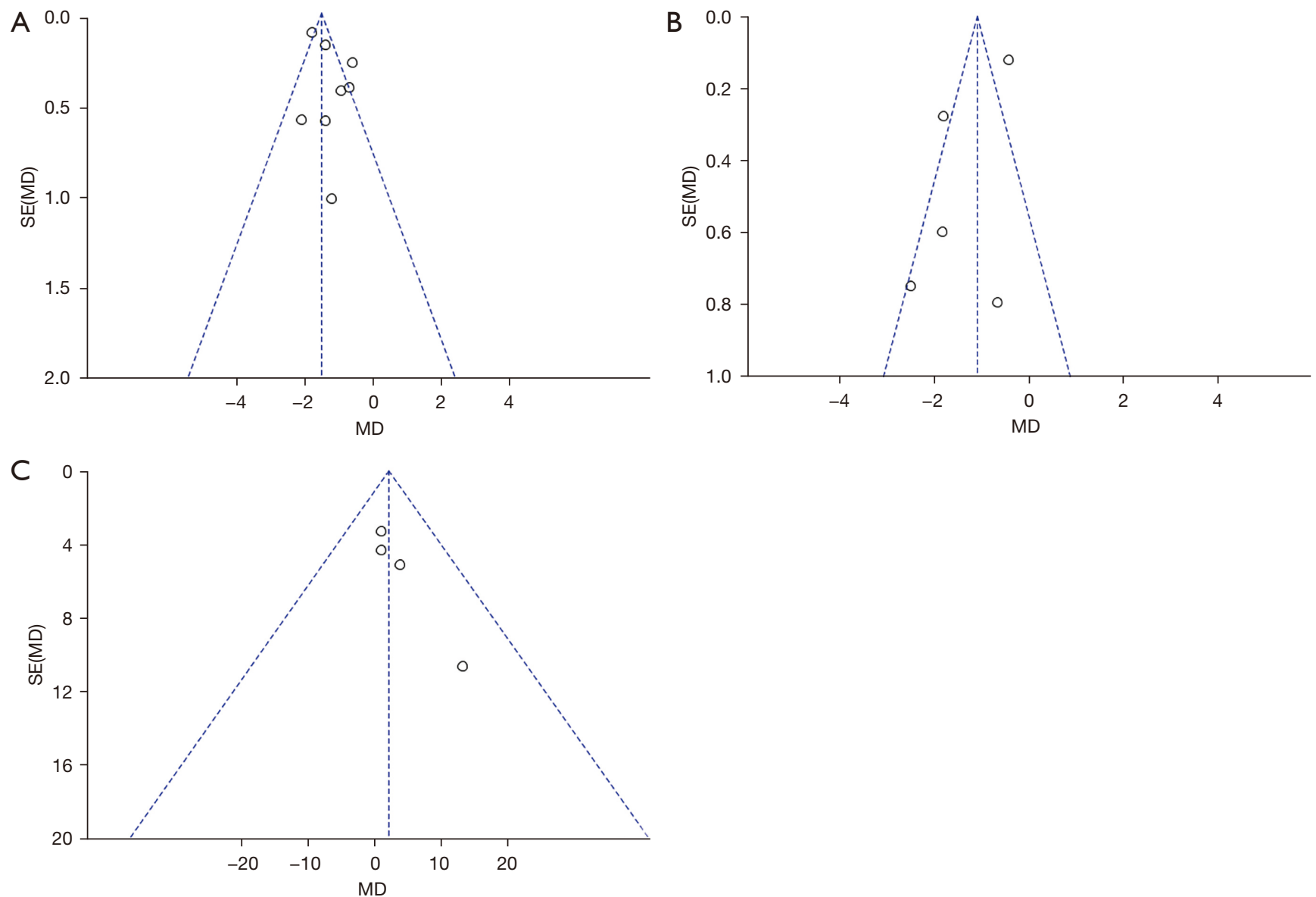

Figure 7 Funnel plots for evaluation indicators (A) for total pain score; (B) for morphine equivalents given in the 24 hours after surgery; (C) for operation duration.

structure was $\mathrm{Z}=0.93(\mathrm{P}=0.35)$. These results indicated that the operation duration scores of the two groups of patients did not differ significantly.

\section{Publication bias analysis}

Publication bias regarding the three evaluation indicators [postoperative pain score (pain), morphine equivalents given in the 24 hours after surgery, and operation duration] of the thoracic nerve group and the control group was also analyzed, with results shown in Figure 7. As can be seen, the funnel plots for the postoperative pain score, the 24-hour dosage of morphine equivalent, and the length of operation are all approximately symmetrical, and the data are also relatively 
concentrated. As seen in Figure $7 B$, only a few samples do not fall within the funnel on the chart. This indicates that there is no significant publication bias regarding the three functional indicators included in studies under review.

\section{Discussion}

In current radical mastectomy surgery, there are still many patients who suffer PMPS, a chronic neuropathic pain syndrome that may occur after mastectomy. The diagnosis of this condition requires the elimination of other causes including long-term pain from preoperative diseases or pain from other external causes, such as malignant tumors and infection. This type of chronic neuropathic pain usually has certain unique characteristics, such as tenderness, loss of sensation, and increasing pain if exacerbated by external factors. This places a serious burden on the physical and psychological well-being of patients who have undergone breast cancer surgery and can significantly affect their postoperative recovery.

The occurrence of PMPS is generally affected by a variety of factors. Because modified radical mastectomy for breast cancer is characterized by excessive cutting and pulling and a large trauma area $(28,29)$, it can cause damage to the thoracic dorsal and intercostal arm nerves. In addition, nerve compression caused by adhesion after surgery may lead to PMPS. External factors such as the patient's age and psychological condition, tissue damage caused by the tumor, and the degeneration of sensory neurons may affect the incidence of PMPS. Currently, there is no clear clinical explanation for the pathogenesis of PMPS. However, relevant data suggest that it is inextricably related to the degree of acute postoperative pain, which, if not correctly managed, is likely to develop into PMPS.

As research into anesthesiologic methods develops, the importance of anesthesia has become apparent not only for the perioperative phase but also for postoperative recovery and patient comfort. With an increasing amount of research being carried out on local nerve blocks in anesthesiology, an abundance of relevant data now shows that a thoracic nerve block can have a good analgesic effect on patients in modified breast cancer surgery. While some related metaanalyses $(30,31)$ have found that a thoracic paravertebral nerve block (TPVB) can significantly reduce the incidence of PMPS, the procedure is relatively complex and can lead to vascular puncture, nerve damage, or other adverse reactions and complications during an operation. Compared with other nerve block methods, thoracic nerve blocks provide the advantages of a simpler, lower-risk, and shorter operation.

In addition to PMPS, postoperative complications such as nausea and vomiting can seriously affect a patient's postoperative comfort and recovery progress. The incidence of postoperative nausea and vomiting is generally correlated to the degree of pain a patient suffers $(32,33)$. Opioid analgesics can effectively relieve postoperative pain but will increase the incidence of postoperative nausea and vomiting. In addition, postoperative vomiting often brings greater pain to patients, and violent vomiting may lead to the surgical wound opening and can affect the patient's ability to take food and medication, which will, in turn, have a serious impact on their postoperative recovery. However, the bone markers need to be clarified before the nerve block puncture, and the dose of local anesthetics needs to be strictly controlled. It is necessary to control the depth of the puncture needle during operation to prevent pneumothorax from puncturing the pleura. At the same time, it is necessary to repeatedly suck back before injecting the drug to avoid local anesthetic drug poisoning.

In summary, this study analyzed and compared the efficacy of a thoracic nerve block in modified radical mastectomy for breast cancer. The results show that a thoracic nerve block can effectively reduce both the amount of morphine used during surgery and the postoperative pain of patients. However, due to the limited number of evaluation indicators in this study, further research with a larger number of indicators is recommended.

\section{Conclusions}

In all, 8 suitable references comprising 624 patients were selected to explore the effect of general anesthesia combined with a thoracic nerve block in modified breast cancer surgery. Outcome indicators were the postoperative pain score, postoperative morphine equivalents given in the 24 hours after surgery, and operation duration. The Jadad tool and the Cochrane Handbook for Systematic Reviews of Interventions were used to evaluate the quality of the selected studies, and RevMan 5.3 was used to conduct a meta-analysis of the experimental data. According to the results of the meta-analysis, the total amount of morphine equivalents in the thoracic nerve group and the control group was heterogeneous $\left(\mathrm{I}^{2}=98 \% ; \mathrm{P}=0.02\right)$. The $\mathrm{MD}$ of the total pain score was -1.27 (95\% CI: -1.68 to -0.86 ), and the statistical test result was $\mathrm{Z}=6.08(\mathrm{P}<0.00001)$. The MD for operation duration was 2.11 (95\% CI: -2.35 to 6.58), and the statistical test result was $\mathrm{Z}=0.93(\mathrm{P}=0.35)$. In 
summary, the results show that general anesthesia combined with a thoracic nerve block can effectively improve the postoperative pain of patients with breast cancer surgery and effectively reduce the use of analgesics. However, the outcome indicators included in this study are insufficient, and it is necessary to increase the sample size and number of outcome indicators to provide further theoretical evidence for the subsequent application of thoracic nerve blocks in modified breast cancer surgery.

\section{Acknowledgments}

Funding: This study received funding from the Science and Technology Plan Project of Sichuan Province, China (No. 2019YJS0221 and 2020YJ0452), the Sichuan Provincial People's Hospital Institute Research Fund, China (No. 2017YL18) and the Scientific Research Project of Health Commission of Sichuan Province, China (No. 18PJ160).

\section{Footnote}

Reporting Checklist: The authors have completed the PRISMA reporting checklist. Available at https://dx.doi. org/10.21037/gs-21-719

Conflicts of Interest: All authors have completed the ICMJE uniform disclosure form (available at https://dx.doi. org/10.21037/gs-21-719). The authors have no conflicts of interest to declare.

Ethical Statement: The authors are accountable for all aspects of the work in ensuring that questions related to the accuracy or integrity of any part of the work are appropriately investigated and resolved.

Open Access Statement: This is an Open Access article distributed in accordance with the Creative Commons Attribution-NonCommercial-NoDerivs 4.0 International License (CC BY-NC-ND 4.0), which permits the noncommercial replication and distribution of the article with the strict proviso that no changes or edits are made and the original work is properly cited (including links to both the formal publication through the relevant DOI and the license). See: https://creativecommons.org/licenses/by-nc-nd/4.0/.

\section{References}

1. Slepicka PF, Cyrill SL, Dos Santos CO. Pregnancy and breast cancer: pathways to understand risk and prevention. Trends Mol Med 2019;25:866-81.

2. Ye LJ, Suo HD, Liang CY, et al. Nomogram for predicting the risk of bone metastasis in breast cancer: a SEER population-based study. Transl Cancer Res 2020;9:6710-9.

3. Seely JM, Alhassan T. Screening for breast cancer in 2018-what should we be doing today? Curr Oncol 2018;25:S115-24.

4. Larsson IM, Ahm Sørensen J, Bille C. The postmastectomy pain syndrome-a systematic review of the treatment modalities. Breast J 2017;23:338-43.

5. Tait RC, Zoberi K, Ferguson M, et al. Persistent postmastectomy pain: risk factors and current approaches to treatment. J Pain 2018;19:1367-83.

6. Abbas DN, Reyad RM. Thermal versus super voltage pulsed radiofrequency of stellate ganglion in postmastectomy neuropathic pain syndrome: a prospective randomized trial. Pain Physician 2018;21:351-62.

7. Wang K, Yee C, Tam S, et al. Prevalence of pain in patients with breast cancer post-treatment: A systematic review. Breast 2018;42:113-27.

8. Chappell AG, Bai J, Yuksel S, et al. Post-mastectomy pain syndrome: defining perioperative etiologies to guide new methods of prevention for plastic surgeons. World J Plast Surg 2020;9:247-53.

9. Ayandipo OO, Adigun TA, Afuwape OO, et al. Comparison of postoperative pain in diathermy and conventional scalpel Skin incision after mastectomy in Ibadan, Nigeria. Afr J Med Med Sci 2015;44:27-31.

10. Couceiro TC, Valença MM, Raposo MC, et al. Prevalence of post-mastectomy pain syndrome and associated risk factors: a cross-sectional cohort study. Pain Manag Nurs 2014;15:731-7.

11. Hasoon J, Urits I, Viswanath O, et al. Erector spinae plane block for the treatment of post mastectomy pain syndrome. Cureus 2021;13:e12656.

12. Urits I, Lavin C, Patel M, et al. Chronic pain following cosmetic breast surgery: a comprehensive review. Pain Ther 2020;9:71-82.

13. Salman AS, Abbas DN, Elrawas MM, et al. Postmastectomy pain syndrome after preoperative stellate ganglion block: a randomized controlled trial. Minerva Anestesiol 2021;87:786-93.

14. Woodworth GE, Ivie RMJ, Nelson SM, et al. Perioperative breast analgesia: a qualitative review of anatomy and regional techniques. Reg Anesth Pain Med 2017;42:609-31.

15. Altıparmak B, Korkmaz Toker M, Uysal AI, et al. 
Evaluation of ultrasound-guided rhomboid intercostal nerve block for postoperative analgesia in breast cancer surgery: a prospective, randomized controlled trial. Reg Anesth Pain Med 2020;45:277-82.

16. Versyck B, van Geffen GJ, Chin KJ. Analgesic efficacy of the Pecs II block: a systematic review and meta-analysis. Anaesthesia 2019;74:663-73.

17. Martsiniv VV, Loskutov AO, Strokan MA, et al. Efficacy of pectoral nerve block type II versus thoracic paravertebral block for analgesia in breast cancer surgery. Klin Onkol 2020;33:296-301.

18. Cui X, Zhu C, Chen P, et al. Effect of pectoral nerve block type II under general anesthesia on the immune function of patients with breast cancer. Am J Surg 2020;220:938-44.

19. Kubodera K, Fujii T, Akane A, et al. <Editors' Choice> Efficacy of pectoral nerve block type-2 (Pecs II block) versus serratus plane block for postoperative analgesia in breast cancer surgery: a retrospective study. Nagoya J Med Sci 2020;82:93-9.

20. Matsumoto M, Flores EM, Kimachi PP, et al. Benefits in radical mastectomy protocol: a randomized trial evaluating the use of regional anesthesia. Sci Rep 2018;8:7815.

21. Bashandy GM, Abbas DN. Pectoral nerves I and II blocks in multimodal analgesia for breast cancer surgery: a randomized clinical trial. Reg Anesth Pain Med 2015;40:68-74.

22. Cros J, Sengès P, Kaprelian S, et al. Pectoral I block does not improve postoperative analgesia after breast cancer surgery: a randomized, double-blind, dual-centered controlled trial. Reg Anesth Pain Med 2018;43:596-604.

23. Kamiya Y, Hasegawa M, Yoshida T, et al. Impact of pectoral nerve block on postoperative pain and quality of recovery in patients undergoing breast cancer surgery: A randomised controlled trial. Eur J Anaesthesiol 2018;35:215-23.

24. Lanier ST, Lewis KC, Kendall MC, et al. Intraoperative nerve blocks fail to improve quality of recovery after tissue expander breast reconstruction: a prospective, doubleblinded, randomized, placebo-controlled clinical trial. Plast Reconstr Surg 2018;141:590-7.

25. Kim DH, Kim S, Kim CS, et al. Efficacy of pectoral nerve

Cite this article as: Liao J, Li M, Gan J, Xiao J, Xiang G, Ding X, Jiang R, Li P. Systematic review and meta-analysis of the efficacy of general anesthesia combined with a thoracic nerve block in modified breast cancer surgery. Gland Surg 2021;10(11):3106-3115. doi: 10.21037/gs-21-719 block type II for breast-conserving surgery and sentinel lymph node biopsy: a prospective randomized controlled study. Pain Res Manag 2018;2018:4315931.

26. M N, Pandey RK, Sharma A, et al. Pectoral nerve blocks to improve analgesia after breast cancer surgery: a prospective, randomized and controlled trial. J Clin Anesth 2018;45:12-7.

27. Versyck B, van Geffen GJ, Van Houwe P. Prospective double blind randomized placebo-controlled clinical trial of the pectoral nerves (Pecs) block type II. J Clin Anesth 2017;40:46-50.

28. Altıparmak B, Korkmaz Toker M, Uysal Aİ, et al. Comparison of the effects of modified pectoral nerve block and erector spinae plane block on postoperative opioid consumption and pain scores of patients after radical mastectomy surgery: A prospective, randomized, controlled trial. J Clin Anesth 2019;54:61-5.

29. Gad M, Abdelwahab K, Abdallah A, et al. Ultrasoundguided erector spinae plane block compared to modified pectoral plane block for modified radical mastectomy operations. Anesth Essays Res 2019;13:334-9.

30. Abu Elyazed MM, Abdelghany MS, Mostafa SF. The analgesic efficacy of pecto-intercostal fascial block combined with pectoral nerve block in modified radical mastectomy: a prospective randomized trial. Pain Physician 2020;23:485-93.

31. Debbag S, Saricaoglu F. Pectoral nerve block as the sole anesthetic technique for a modified radical mastectomy in a comorbid patient. Saudi Med J 2019;40:1285-9.

32. Jiménez-Tornero J, Cortés-Flores AO, Chávez-Tostado $M$, et al. Effect of a preoperative single-dose steroid on pulmonary function and postoperative symptoms after modified radical mastectomy: results of a randomized clinical trial. Gland Surg 2020;9:1313-27.

33. Bakeer AH, Kamel KM, Abdelgalil AS, et al. Modified pectoral nerve block versus serratus block for analgesia following modified radical mastectomy: a randomized controlled trial. J Pain Res 2020;13:1769-75.

(English Language Editors: L. Roberts and J. Gray) 